\title{
Scoping clinicians' perspectives on pre-treatment multidisciplinary care for young women with breast cancer
}

\author{
Arden L Corter' \\ May Lynn Quan² \\ Frances L Wright ${ }^{3}$ \\ Erin D Kennedy ${ }^{4}$ \\ Marko RI Simunovic ${ }^{5}$ \\ Juliet Shao' \\ Nancy N Baxter ${ }^{1,6}$ \\ 'Department of Surgery, Li Ka \\ Shing Knowledge Institute, St. \\ Michael's Hospital, Toronto, Canada; \\ ${ }^{2}$ Department of Surgery and \\ Oncology, University of Calgary, \\ Calgary, Canada; ${ }^{3}$ Department of \\ Surgery, University of Toronto, \\ Toronto, Canada; ${ }^{4}$ Division of General \\ Surgery, University Health Network, \\ Mount Sinai Hospital, Toronto, \\ Canada; ${ }^{5}$ Department of Surgery, \\ McMaster University, Hamilton, \\ Canada; ${ }^{6}$ Dalla Lana School of Public \\ Health, University of Toronto, Toronto, \\ Canada
}

This article was published in the following Dove Press journal: Journal of Multidisciplinary Healthcare

Background: Young women with breast cancer (YWBC) experience worse medical and psychosocial outcomes than their older counterparts. Early input from a multidisciplinary team via pre-treatment multidisciplinary cancer conferences (pMCCs) may be important for addressing the complex needs of YWBC. However, pMCCs are not common. This study has two parts: a survey and workshop aimed at assessing clinicians' perspectives on pMCCs, including the importance of pMCCs in the care of YWBC, as well as barriers to, and strategies for supporting their implementation.

Methods: Survey results highlight variability across sites in the delivery of multidisciplinary care in general. However, both survey and workshop results emphasize clinicians' agreement on the importance of pMCCs and suggest that numerous practical and systems levels barriers be addressed before pMCCs can be implemented.

Conclusions: pMCCs have the potential to improve surgical treatment and psychosocial outcomes for YWBC. A combined practical and policy approach to their implementation, which sees extension of existing standards to include pMCCs, may support their adoption and subsequent audit practices to assess the effect of pMCCs on outcomes for YWBC.

Keywords: multidisciplinary care, pre-treatment, cancer conference, breast cancer, young women

\section{Background}

Young women $(<45$ years) with breast cancer (YWBC) have complex healthcare needs. They are more likely to be diagnosed with aggressive forms of cancer, ${ }^{1,2}$ have higher surgical re-excision rates ${ }^{3}$ and have greater risk of cancer recurrence and mortality than older women. ${ }^{4,5}$ Fertility concerns may also be an issue, as YWBC may be pregnant at the time of diagnosis or, may be looking forward to having children in the future. ${ }^{6,7}$ Furthermore, the greater likelihood of genetic contributions to the diagnosis ${ }^{8}$ may contribute to concerns about passing cancer genes onto children or to concerns about cancer recurrence. General psychosocial outcomes are also worse. YWBC are at higher risk of depression and anxiety, which in turn affect quality of life. ${ }^{9}$ Financial, ${ }^{10}$ career,${ }^{11}$ parenting, ${ }^{12}$ body image and sexuality concerns ${ }^{13}$ may also contribute to poorer adjustment.

Early input from a multidisciplinary team may be important for identifying and Correspondence: Nancy N Baxter St. Michael's Hospital, 16 CC-040, 30 Bond Street, Toronto, Ontario, M5BIW8, Canada

$\mathrm{Tel}+\mathrm{I} 4168645168$

$\mathrm{Fax}+\mathrm{I} 4163600637$

Email baxtern@smh.ca addressing the mix of treatment and psychosocial issues YWBC face, and for supporting care coordination, including timely referral to treatment and support services. Multidisciplinary cancer conferences (MCCs) are regularly scheduled meetings where medical and other health professionals prospectively discuss appropriate diagnostic 
tests and suitable treatment options for individual patients. ${ }^{14}$ Research points to the benefits of multidisciplinary care/ $\mathrm{MCCs}^{15}$ including: more accurate diagnosis, and improved concordance with treatment guidelines ${ }^{16}$; improved quality and efficiency of inter-professional communication; ongoing professional education ${ }^{17}$; and discussion of patients eligible for novel/trial treatments. ${ }^{18,19}$

Pre-treatment MCCs (pMCCs) may add additional benefit for treatment decisions and care of YWBC. For example, in a study of 149 breast cancer patients seeking second opinions, Newman et al found that recommendations for surgical management changed in $52 \%$ of patients presented to pre/ post MCC at a comprehensive care center, suggesting that earlier presentation may support treatment recommendation. ${ }^{20}$ Recent guidelines aimed at reducing lumpectomy reoperations in breast cancer patients recommend pre-treatment multidisciplinary care including radiologists, surgeons, pathologists and medical oncologists. ${ }^{21}$ Despite the promise of pMCCs in supporting cancer care for YWBC, research indicates that $\mathrm{pMCC}$ are rare. ${ }^{22}$ Furthermore, fertility support for YWBC falls short of recommendations for care. ${ }^{23}$

The present research aimed to identify strategies to support pMCC practice for YWBC using the Knowledge to Action (KTA) cycle, a conceptual framework designed to support development and evaluation of health service interventions that meet sector needs and lead to actionable and sustainable changes in health service delivery. ${ }^{24}$ Our research was embedded in a pan-Canadian, prospective cohort study called RUBY: Reducing the burden of breast cancer in young women. Our objectives were to support pMCC practice by understanding the implementation context through two related studies: 1) a survey of practitioners' perspectives on local multidisciplinary practice and opportunities for enhancing multidisciplinary care of YWBC at RUBY recruiting sites; and 2) a key informant workshop with RUBY site leads and co-investigators to identify strategies for improving multidisciplinary care of YWBC. Approval for the study was granted by St Michael's Hospital Research Ethics Board (REB\# 14-309C).

\section{Part I: Practitioner survey}

This survey aimed to obtain broad feedback on perceptions of current multidisciplinary care practices of YWBC, and to identify perceptions of, and opportunities for, implementing pMCCs.

\section{Methods}

\section{Participants and setting}

RUBY sites represented 10 Canadian provinces and 29 recruiting sites. Each site has a designated surgeon investigator who serves as site lead. For this cross-sectional survey, all site leads received an emailed survey link and were asked to disseminate it to other surgeons, medical and radiation oncologists, pathologists, psychologists, nurses and others involved in the care of YWBC within their site. Consent was implied by opening and clicking through the electronic survey.

\section{Survey instrument}

A 34-item survey assessed participants' perceptions of the importance of pre-treatment consideration of both nonsurgical and surgical issues when planning for the care and treatment for YWBC (eg, multidisciplinary consideration of neoadjuvant treatment; and multidisciplinary assessment of the appropriateness of breast conserving therapy). Other questions related to the possible utility of strategies for optimizing pre-treatment management (eg, routine use of pre-treatment checklists) and perceptions of the necessity of representatives from different professional groups attending pMCCs. Respondents rated all questions on 5-point Likert scales from 1 (not at all important/never) to 5 (extremely important/always). Open-ended questions asked participants to describe current care coordination practices within their institution, desired improvements in multidisciplinary care, and barriers to and facilitators of pMCCs.

\section{Procedure}

The survey was developed using Survey Monkey ${ }^{\mathrm{TM}}$ and distributed via email between March-April 2015. A reminder email followed, 1 week after initial distribution.

\section{Data analysis}

Analysis included descriptive statistics for each numerical survey question. Qualitative survey data were analyzed using a general inductive approach, which involves a detailed review of data, and identification and categorization of emerging themes in an iterative approach until all themes can be categorized. ${ }^{25}$ Independent coders (JS \& AC) reviewed data, and any disagreements were discussed and resolved.

\section{Results}

Ninety health professionals responded to the survey, including 39 surgeons, 11 nurses, 11 medical oncologists, ten radiation oncologists, five pathologists, five radiologists, four "other" (ie, family physician, palliative care physician, social worker and patient navigator), and five unspecified.

Table 1 shows respondents' ratings of the importance of non-surgical issues in the pre-treatment care of YWBC. 
Highest ratings were for multidisciplinary consideration of neoadjuvant treatment $(\mathrm{M}=4.7 ; \mathrm{SD}=0.5)$, followed by assessment of fertility preservation $(\mathrm{M}=4.5 ; \mathrm{SD}=0.7)$, and psychosocial counseling needs $(\mathrm{M}=4.5 ; \mathrm{SD}=0.7)$, evaluation of genetic predisposition risk $(\mathrm{M}=4.4 ; \mathrm{SD}=0.7)$, patient navigation $(M=4.4 ; S D=0.7)$, referral to fertility specialist $(\mathrm{M}=4.2 ; \mathrm{SD}=0.8)$, and pre-treatment consultation with radiation oncologist $(\mathrm{M}=3.6, \mathrm{SD}=1.0)$. Ratings for surgical issues in the overall care of YWBC (Table 2), were highest for multidisciplinary assessment of appropriateness of breast conserving therapy $(\mathrm{M}=4.3 ; \mathrm{SD}=0.8)$, consideration of immediate breast reconstruction $(\mathrm{M}=4.2 ; \mathrm{SD}=0.7)$, and pre-treatment multidisciplinary review of breast imaging $(\mathrm{M}=4.1 ; \mathrm{SD}=0.8)$.

Ratings of the frequency with which different professional groups would be needed to attend pMCCs are presented in Figure 1. There was strong agreement on the optimal attendance rates for surgeons, treating physicians, medical and radiation oncologists, pathologists and radiologists; the majority of participants agreed that these groups should attend "always" to "half of the time." More varied perceptions existed on optimal attendance for nursing staff, reconstructive surgeons, geneticists, social workers and fertility specialists.
An open-ended question asked respondents how care for YWBC was currently coordinated. Of the $74(82 \%)$ who answered this question, only a third $(n=24)$ indicated that MCCs (pre or post) were part of routine practice, and many described care coordination as ad hoc, or conducted on an "as needed" and informal basis. There were variable reports on the timing of multidisciplinary care as pre- or postoperative or both pre- and postoperative.

Sixty-six participants $(73 \%)$ reported on one or more barriers to implementing pMCCs for YWBC. The most commonly reported was time (eg, time to attend and coordinate meetings) $(\mathrm{n}=19,29 \%)$, followed by lack of resources (eg, money, clinic space, IT supports) ( $\mathrm{n}=17 ; 26 \%)$, service access (eg, to genetics and diagnostic testing, to medical equipment and specialists) ( $\mathrm{n}=16 ; 24 \%$ ), and report delays (eg, pathology reports) $(\mathrm{n}=12 ; 18 \%)$. Other barriers included problematic professional participation (eg, resistance to team approach, lack of interest) $(\mathrm{n}=8 ; 12 \%)$; challenges with team coordination (eg, liaising with professionals within and across sites) $(\mathrm{n}=7 ; 11 \%)$; and lack of formal processes to guide pMCCs for YWBC $(\mathrm{n}=3 ; 5 \%)$.

Sixty participants (67\%) commented on facilitators that would support pMCCs. These included staff support (eg,

Table I Participants' ratings of the importance of non-surgical and surgical issues in the care of YWBC

\begin{tabular}{|c|c|c|c|}
\hline $\begin{array}{l}\text { Importance of nonsurgical } \\
\text { pre-treatment issues }\end{array}$ & $\begin{array}{l}\text { Somewhat } \\
\text { important to } \\
\text { extremely } \\
\text { important n (\%) }\end{array}$ & $\begin{array}{l}\text { Neutral } \\
\text { n (\%) }\end{array}$ & $\begin{array}{l}\text { Not at all important } \\
\text { to not that } \\
\text { important } n(\%)\end{array}$ \\
\hline Multi-disciplinary consideration of neoadjuvant treatment (chemotherapy, radiation) & $89(96.0)$ & $3(3.2)$ & $0(0)$ \\
\hline Assessment of fertility preservation needs & $87(93.5)$ & $3(3.2)$ & $2(2.2)$ \\
\hline Assessment of psychosocial counseling needs & $83(89.2)$ & $8(8.6)$ & $\mathrm{I}(\mathrm{I} . \mathrm{I})$ \\
\hline Evaluation of genetic predisposition risk & $83(89.2)$ & $8(8.6)$ & I (I.I) \\
\hline Patient navigation & $83(89.2)$ & $8(8.6)$ & $\mathrm{I}(\mathrm{I} . \mathrm{I})$ \\
\hline Pre-treatment referral to fertility specialist & $76(81.7)$ & $13(14.0)$ & $2(2.2)$ \\
\hline Pre-treatment radiation oncology consultation & $52(56.0)$ & $26(28.0)$ & $13(14)$ \\
\hline \multicolumn{4}{|l|}{ Importance of surgical issues in the overall care of YWBC } \\
\hline Multi-disciplinary assessment of appropriateness of breast conserving therapy & $82(88.1)$ & $7(7.5)$ & $3(3.2)$ \\
\hline Consideration of immediate breast reconstruction & $79(84.9$ & $10(10.6)$ & $3(3.2)$ \\
\hline Pre-treatment multi-disciplinary review of breast imaging & $75(80.6)$ & $12(12.9)$ & $4(4.3)$ \\
\hline Consideration of contralateral prophylactic mastectomy & $55(59.1)$ & $25(26.9)$ & $10(10.6)$ \\
\hline
\end{tabular}

Notes: Survey responses have been collapsed into a 3-point scale from the original 5-point scale.

Abbreviations: YWBC, young women with breast cancer.

Table 2 Participants' ratings of usefulness of pre-treatment strategies for management of YWBC

\begin{tabular}{llll}
\hline $\begin{array}{l}\text { Pre-treatment } \\
\text { strategy }\end{array}$ & $\begin{array}{l}\text { Somewhat useful to } \\
\text { extremely useful } \mathbf{n}(\%)\end{array}$ & $\begin{array}{l}\text { Neutral } \\
\mathbf{n}(\%)\end{array}$ & $\begin{array}{l}\text { Not at all useful to } \\
\text { not that useful } \mathbf{n}(\%)\end{array}$ \\
\hline pMCC of cases treated at your site & $72(80)$ & $12(13)$ & $2(2)$ \\
Routine use of pre-treatment checklists & $53(59)$ & $10(2 I)$ & $12(13)$ \\
Ad hoc multidisciplinary review pre-treatment & $46(5 I)$ & $29(5 I)$ & $8(9)$ \\
Pre-treatment multidisciplinary review via secure email & $43(48)$ & $28(3 I)$ & $14(16)$ \\
\hline
\end{tabular}

Notes: Survey responses have been collapsed into a 3-point scale from the original 5-point scale.

Abbreviations: pMCCs, pre-treatment multidisciplinary cancer conferences; YWBC, young women with breast cancer. 


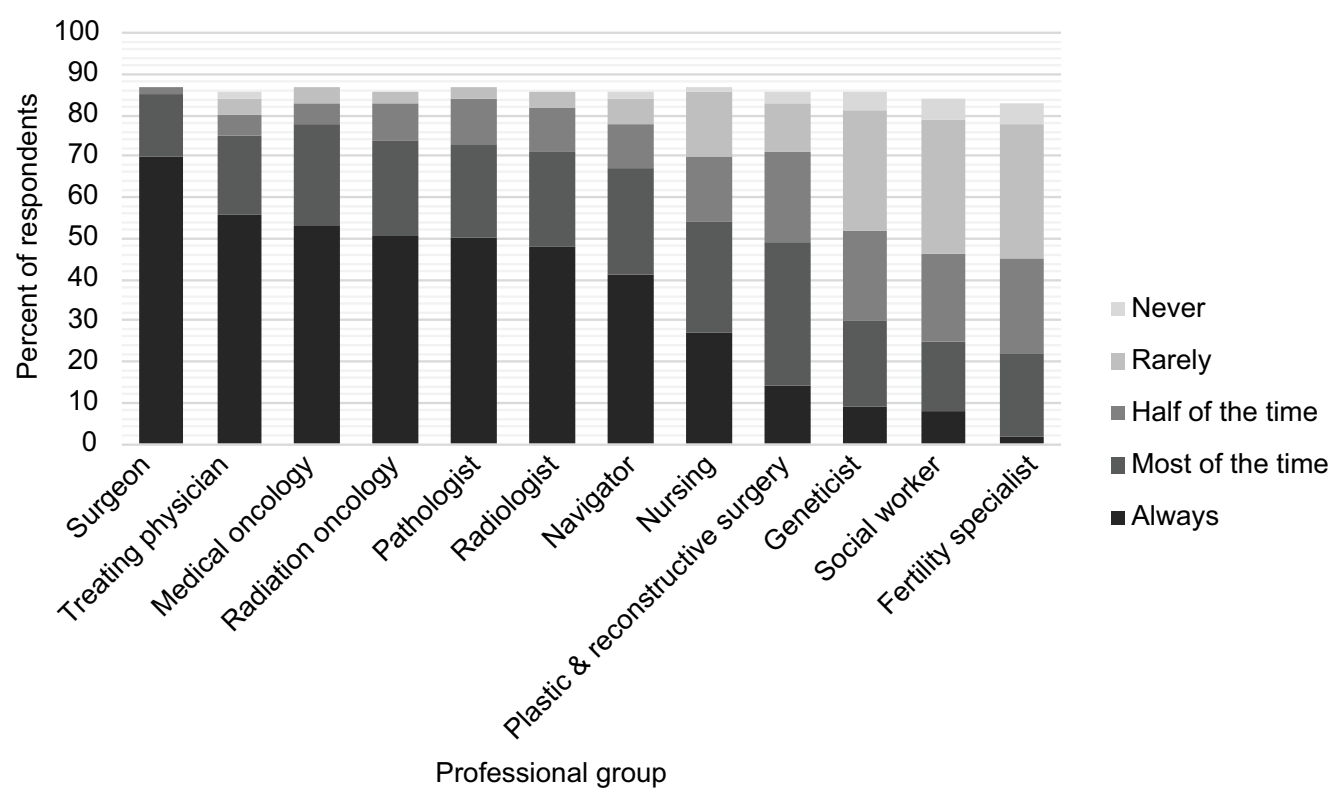

Figure I Ratings of the frequency with which specialist groups should attend multidisciplinary cancer conferences.

administrative staff, nurse specialists, patient navigators) $(\mathrm{n}=19 ; 32 \%)$; implementation of structured pMCCs $(\mathrm{n}=13$; $22 \%)$; quality leadership to champion pMCCs $(n=7,12 \%)$; and tools/standards to support meetings (eg, expedited or common care pathways for YWBC, national standards) $(n=6 ; 10 \%)$. Other facilitators listed by four or fewer participants included: high functioning teams who appreciate each other's roles and communicate well; short wait times for pre-treatment tests and reports; comprehensive cancer centers/clinics; care coordination; and resources such as patient support, and incentives to attend rounds.

Ninety-seven percent of question respondents (66 of 68 ) reported that improvements in multidisciplinary care were needed. Most commonly, these included formalizing processes for the delivery of multidisciplinary care $(n=28$; $41 \%$ ). For example, participants suggested implementing pMCCs for treatment planning, increasing the frequency of multidisciplinary meetings; ensuring that $\mathrm{pMCCs}$ are routine for YWBC; and incorporating telemedicine to support delivery of multidisciplinary care. Fourteen participants $(21 \%)$ commented on the need for improvements in the consistency and representation of professional participation in multidisciplinary team meetings, and 14 (21\%) mentioned the need for better and more timely service access (eg, to fertility services, reconstructive surgeons, psychosocial support, diagnostic testing). Improved coordination of care including improvements in referral processes was also mentioned $(n=11 ; 16 \%)$.

\section{Part 2: Key informant workshop}

The purpose of the key informant workshop was to delve more deeply into clinicians' perspectives on strategies for improving multidisciplinary care ofYWBC, and the context for supporting implementation of pMCCs across RUBY sites.

\section{Methods}

\section{Participants and setting}

Participants in the key informant workshop were 39 RUBY investigators from rural, urban, academic and community settings across Canada attending a pre-existing RUBY conference. Participants included surgical oncologists $(n=17)$, surgeons $(n=12)$, research scientists $(n=5)$, medical directors $(\mathrm{n}=2)$, a radiologist $(\mathrm{n}=1)$, and medical oncologist $(\mathrm{n}=1)$, and represented 35 research/treatment facilities across eight provinces/territories: Alberta, British Columbia, New Brunswick, Manitoba, Ontario, Quebec, Saskatchewan, Yukon. The one-day workshop took place in Toronto, in April 2015.

\section{Materials}

Two break-out sessions were held, with participants assigned to groups and asked to address three topics in the morning session and two topics in the afternoon. Morning session topics were: 1) What model to improve multidisciplinary care would work at your institution? 2) What are the advantages and disadvantages to the model options, and, which one do you favor? 3) What strategies could be used to implement 
the pMCC model at your centers? Afternoon session topics were: 4) What management elements are the most important and need to be addressed pre-treatment for YWBC? and 5) What quality indicators should be used to measure success of pre-treatment multidisciplinary care?

Following these sessions, attendees voted agree/disagree on key issues in the multidisciplinary care of YWBC including: 1) ideally, the best model to pursue multidisciplinary care is a case conference with identified specialists in the same room or in virtual attendance; 2) ideally, RUBY eligible patients will be presented at pMCCs; 3 ) ideally, consecutive (ie, all) RUBY eligible patients will be presented at pMCCs; and 4) ideally, at a minimum, the following six specialties should be present at the pMCC: surgeon, radiation oncologist, medical oncologist, radiologist, pathologist and reconstructive surgeon.

\section{Procedure}

Prior to the break-out sessions, participants attended a presentation on RUBY's Knowledge to Action workstream: Improving local therapy for young women across Canada. This presentation oriented all attendees to issues in the care of YWBC and in pMCCs and served to preface the workshop's goals. Break-out sessions were then held with approximately ten participants per group. Each session lasted roughly 1.5 hours and was facilitated by RUBY research coordinators. Following the morning and afternoon sessions, a 30-minute large-group discussion and synthesis of findings was conducted, and the vote on key issues in the multidisciplinary care of YWBC was conducted. Detailed notes were taken of all group sessions, then summarized by research assistants.

\section{Analysis}

Consistent with methods used to evaluate qualitative survey data, a general inductive approach was used to categorize themes with two independent coders (JC \& AC).

\section{Results}

In response to question one, most attendees reported favoring the pMCC model compared with other forms of multidisciplinary/ad hoc care. Table 3 presents facets of the model considered important, as well as key themes and details identified from other workshop questions (\#2-5).

Workshop participants also voted on whether they agreed or disagreed with issues related to the care of YWBC. The results are presented in Table 4. Overall, they neared perfect consensus on pMCC with consecutive presentation considered the ideal form of multidisciplinary care of YWBC.
There was less consensus on the types of professionals who should attend pMCCs.

\section{Discussion}

This study assessed practitioners' perspectives on current multidisciplinary care practices for YWBC and their perceptions of possibilities for pMCCs, including which treatment issues would be appropriate for consideration, which professionals should attend, and what barriers, facilitators, and strategies exist in implementation.

\section{Current MCC practice}

The broad survey of practitioners revealed that some sort of multidisciplinary care via tumor board or clinic rounds existed in their sites. However, more than half reported sporadic or ad hoc multidisciplinary care. Most respondents indicated that improvements in multidisciplinary care are needed such as formalizing processes of the functioning of pMCCs, ensuring routine pMCC of YWBC and improving the consistency and representation of professional participation in multidisciplinary team meetings. This picture of current practice indicates significant practice gaps given evidence for the benefits of MCCs, ${ }^{18}$ the existence of standards to guide practice, and the perspective of equitable patient access to quality care. These gaps are not unique to the sites surveyed in this study. ${ }^{26,27}$ The data in this study point to ways in which pMCCs might be implemented, and KTA cycles used to guide implementation of pMCCs as a potential solution to gaps in practice.

\section{Issues for consideration in PMCCs}

Participants gave high ratings to the importance of a variety of nonsurgical issues in the pre-treatment management of YWBC, such as psychosocial support and fertility preservation. Agreement was somewhat lower on ratings of the importance of pre-treatment referrals to fertility specialists and radiation oncologists, suggesting that conferencing on these needs in pMCCs might be a better model than automatic pre-treatment referrals. There was also general agreement on the importance of surgical issues for pre-treatment consideration. The exception was that consideration of contralateral prophylactic mastectomy was seen as less important. This may be due to mixed evidence for prophylactic mastectomy, including some research showing no survival benefits. ${ }^{28,29}$ Nevertheless, there was near consensus that a variety of non-surgical and surgical needs should be considered pretreatment. The variety of these needs, ranging from psychosocial to breast conserving treatment, and the fact that many 
Table 3 Key themes and details from attendees' responses to workshop questions

\begin{tabular}{|c|c|c|c|}
\hline \# & Workshop question & Key theme & Details \\
\hline \multirow[t]{4}{*}{ I } & Important facets of the & Timing & Meetings routinely scheduled to suit as many disciplines as possible \\
\hline & pMCC model? & Attendance & $\begin{array}{l}\text { Meetings attended at least by a surgeon, medical oncologist, radiation oncologist, } \\
\text { pathologist and radiologist }\end{array}$ \\
\hline & & Patient outcomes & Recommendations for patient management made and documented \\
\hline & & IT support & Video and teleconferencing technology available to enable virtual attendance \\
\hline $2 \mathrm{a}$ & $\begin{array}{l}\text { Advantages of the pMCC } \\
\text { model? }\end{array}$ & Existing resources & The ability to build on existing resources (eg, existing networks, clinical resources) \\
\hline \multirow[t]{5}{*}{$2 b$} & Disadvantages of the & Low attendance & Key disciplines not attending even with appropriately timed meetings \\
\hline & pMCC model? & Lack of resources & Insufficient resource to accommodate and support meetings \\
\hline & & No accreditation & Processes involved in implementing \& operating PMCCs not recognized \\
\hline & & No mandate & Expectations to implement and monitor PMCCs not set out \\
\hline & & No remuneration & Attendance at additional meetings not funded \\
\hline \multirow[t]{6}{*}{$3 a$} & $\begin{array}{l}\text { Strategies that could be } \\
\text { used to implement the } \\
\text { pMCC model? }\end{array}$ & Champions/leadership & To guide implementation and sustainability \\
\hline & & Pre-treatment checklists/ & To support appropriate referrals and attendance, and to help document pMCCs \\
\hline & & Templates & outcomes, as well as prepopulated templates to reduce documentation load \\
\hline & & Clinical education & To raise awareness about the benefits of presenting YWBC at MCCs \\
\hline & & pMCC Chairperson & To direct the flow of pMCCs \\
\hline & & Accessible meeting times & To support attendance \\
\hline \multirow[t]{5}{*}{4} & Important management & Diagnosis & Confirmation of diagnosis and stage \\
\hline & $\begin{array}{l}\text { elements for PMCC care } \\
\text { of YWBC? }\end{array}$ & Surgical plan & $\begin{array}{l}\text { Decision making re: breast conservation or mastectomy; reconstruction or no } \\
\text { reconstruction }\end{array}$ \\
\hline & & Reports & Review imaging \& pathology \\
\hline & & (Neo)adjuvant plan & Oncology \& radiation plan considered \\
\hline & & Psychosocial concerns & Address psychosocial concerns through assessment \& referral \\
\hline \multirow[t]{8}{*}{5} & $\begin{array}{l}\text { Quality indicators to } \\
\text { measure the success of }\end{array}$ & Professional attendance & $\begin{array}{l}\text { Attendance at a minimum by surgery, radiology, radiation oncology, pathology, medical } \\
\text { oncology and reconstructive surgery }\end{array}$ \\
\hline & pMCCs? & Presentation & The proportion of YWBC presented to pMCCs \\
\hline & & Referrals & The proportion of women being offered and/or receiving pre-treatment referrals \\
\hline & & Time & $\begin{array}{l}\text { Time from initial consultation to first treatment to assess unintended consequences } \\
\text { (eg, delay in care from a wait for } \mathrm{PMCC} \text { ). }\end{array}$ \\
\hline & & Treatment plan & $\begin{array}{l}\text { Changes in plans resulting from pMCC (eg, change from preliminary plan after } \\
\text { recommendation from } \mathrm{MCC} \text { ) }\end{array}$ \\
\hline & & Adherence & Whether PMCC treatment plan recommendation was followed \\
\hline & & Checklist completion & The number of completed pre-treatment checklists \\
\hline & & Satisfaction & Patient and provider ratings of satisfaction with PMCC recommendations \\
\hline
\end{tabular}

Abbreviations: $M C C(s)$, multidisciplinary cancer conference(s); PMCC(s), pre-treatment multidisciplinary cancer conference(s); YWBC, young women with breast cancer.

Table 4 Proportion of workshop attendees agreeing on key issues in the multidisciplinary care of YWBC

\begin{tabular}{|c|c|c|}
\hline$(\mathbf{N}=\mathbf{2 6})$ & $\begin{array}{l}\text { Agree } \\
\text { N (\%) }\end{array}$ & $\begin{array}{l}\text { Disagree } \\
\text { N (\%) }\end{array}$ \\
\hline $\begin{array}{l}\text { Ideally, the best model to pursue multidisciplinary care is a case conference with identified specialists in the same } \\
\text { room or in virtual attendance. }\end{array}$ & $25(96)$ & I (4) \\
\hline Ideally, RUBY eligible patients will be presented at pMCCs. & $26(100)$ & 0 \\
\hline Ideally, consecutive (ie, all) YWBC will be presented at MCCs. & $26(100)$ & 0 \\
\hline $\begin{array}{l}\text { Ideally, at a minimum, the following six specialties should be represented at MCCs: surgeon, radiation oncologist, } \\
\text { medical oncologist, radiologist, pathologist, plastic surgery. }\end{array}$ & $19(73)$ & $7(27)$ \\
\hline
\end{tabular}

Abbreviations: MCC(s), multidisciplinary cancer conference(s); pMCCs, pretreatment multidisciplinary cancer conference; YWBC, young women with breast cancer.

of the needs explicitly involve multidisciplinary consultation, suggest that pMCCs have the potential to improve practice for YWBC. The KTA cycle requires careful consideration of local and systemic barriers and facilitators, along with other implementation and management issues, in order to realize this potential. 


\section{Barriers}

Barriers to pMCCs identified in the survey and workshop were overlapping and outweighed identified advantages. Many of these barriers echo international research. ${ }^{27,30,31}$ For example, lack of time and issues of funded time were commonly mentioned. In turn, these could affect attendance and attitudes towards meetings. Previous research suggests that failure of attendance of key specialists contributes to less productive meetings. ${ }^{32}$ Other identified barriers related to the practical challenges of implementing pMCCs across diverse treatment settings with varied access to resources.

\section{Facilitators}

Identified facilitators of pMCCs were similar across the workshop and survey and also echoed previous research on facilitators of multidisciplinary care. For example, as in previous research ${ }^{33}$ the need for a clear mandate to support MCCs was identified, as was having more staff support and time to attend meetings. Positive leadership and team dynamics may also support multidisciplinary care ${ }^{17}$ Overall, themes for facilitating pMCCs included processes to improve multidisciplinary care practices in general, and issues for consideration in pMCCs specifically. It is likely that both general and specific process improvements will support change. For example, specific use of checklists to guide pMCC participation and decision-making, and more general administrative processes to support additional multidisciplinary meetings for busy professionals may bolster pMCCs. Indeed, approximately one quarter of survey participants highlighted the need for pMCC care and process standards to support practice.

\section{Implementation and management strategies}

Reporting their perspectives on pMCCs in the workshops, site leads recommended implementation strategies such as practitioner education, having a meeting chairperson, and strategies to coordinate schedules, mirroring those used to support implementation of all forms of MCCs in Ontario. ${ }^{14}$

Participants also generated many management elements for consideration in pMCCs, like reviewing imaging and pathology and confirming diagnoses to support treatment planning. Numerous quality indicators were also identified, including increase in patient referrals, attendance of key professionals, and changes in treatment plans. Currently, there are limited audit processes for the performance of MCCs across Canada. Ontario is the only province with MCC standards $^{34}$ and interventions trialed to support implementation. ${ }^{35}$ However, data on MCCs are not comprehensive. For example, details on attendance records are kept but unpublished, and reports on referral records are unavailable. More in-depth examination of MCC processes and indicators of success as exemplified by audits conducted in other settings ${ }^{36,37}$ may extend our understanding of supports needed to improve multidisciplinary care including pMCCs. In particular, a look at the interactivity of MCCs and the extent and type of inter-professional contribution may inform understanding of where MCCs are functioning on the continuum of multidisciplinary care. Modification of the existing Ontario guidelines to include processes for pMCCs and adaptation of guidelines to other provinces may also support pMCC implementation.

\section{Limitations}

The survey was not a comprehensive stock-take but a scoping of the landscape of current care practices and professional perceptions on pMCCs. We do not know the true extent of variation in MCC or other multidisciplinary cancer care practices at RUBY sites. Furthermore, as the total number of survey recipients is unknown and the survey was anonymous, we do not know which RUBY sites respondents represented. Therefore, results could be biased, if for example, there were more respondents from larger centers, which tend to have better processes in place for multidisciplinary care. Additionally, survey and workshop responses were weighted in favor of surgeons as a result of our sampling strategy, which for the workshop involved opportunistic consultation with attendees at a RUBY investigators' meeting, and for the survey involved snowball sampling through RUBY site leads. As with any survey, it is possible that those more interested in the pMCC model responded to the survey and as a result, our findings are more supportive of that model than they would be given a more mixed sample. Workshop findings are also limited with regard to representativeness given that attendees were already concerned with improving the care of YWBC and mostly work in care centers with established multidisciplinary care practices. Despite these limitations, the research illuminates issues in the implementation and operation of MCCs, including pMCCs. It signals clear opportunities for developing tools to support pMCC practice (eg, clinical checklists), and highlights issues for consideration in future research.

\section{Conclusions and future directions}

Among the first to report on clinicians' perspectives of pMCCs for YWBC, this study provides insights into perceptions of the importance of pMCCs including surgical and nonsurgical management issues, barriers to and facilitators 
of running pMCCs, and strategies for implementing and measuring their success.

Findings indicate that implementation of pMCCs may improve the quality of care for YWBC and that a pathway to introduce and/or support pMCCs should include a multipronged and multi-level approach with:

- Guidelines for the implementation and operation of pMCCs with potential for the adoption of Ontario guidelines,

- Practical supports such as infrastructure for remote and in-person attendance, and checklists to help cue and document clinical care considerations, and

- Staffing and remuneration for attendees, including administrative support, leadership and pMCC champions.

Given findings for the benefits of MCCs in supporting patient care and professional practice, more local and international effort to support ongoing MCC implementation and monitoring is warranted. Future studies should include in-depth examination of variations in multidisciplinary care practice, which would inform development of MCC implementation toolboxes. These efforts would be bolstered by policy work and guidelines to provide targets for practice development and quality audits.

As next steps in the KTA approach, we are conducting a "stock-take" of multidisciplinary care practices across RUBY sites. Based on the Ontario MCC guidelines, the stock-take will provide a better sense of the processes and gaps to be addressed to improve multidisciplinary care across Canadian provinces. Concurrently, we are piloting a checklist aimed at supporting pMCCs for YWBC at RUBY sites.

\section{Acknowledgments}

We would like to thank RUBY study investigators for their time and input. As part of the larger RUBY study, this research was funded through a joint grant from Canadian Institute of Health Research and the Canadian Breast Cancer Foundation (\#01305-000).

\section{Author contributions}

All authors contributed toward data analysis, drafting and critically revising the paper and agree to be accountable for all aspects of the work.

\section{Disclosure}

The authors report no conflicts of interest in this work.

\section{References}

1. Anders CK, Hsu DS, Broadwater G, et al. Young age at diagnosis correlates with worse prognosis and defines a subset of breast cancers with shared patterns of gene expression. J Clin Oncol. 2008;26(20):3324-3330.

2. Kim HJ, Han W, Yi OV, Ov Y, et al. Young age is associated with ipsilateral breast tumor recurrence after breast conserving surgery and radiation therapy in patients with HER2-positive/ER-negative subtype. Breast Cancer Res Treat. 2011;130(2):499-505.

3. Menes TS, Tartter PI, Bleiweiss I, Godbold JH, Estabrook A, Smith SR. The consequence of multiple re-excisions to obtain clear lumpectomy margins in breast cancer patients. Ann Surg Oncol. 2005;12(11):881-885.

4. Fredholm H, Eaker S, Frisell J, Holmberg L, Fredriksson I, Lindman H. Breast cancer in young women: poor survival despite intensive treatment. PLoS One. 2009;4(11):e7695.

5. Han W, Kim SW, Park IA, et al. Young age: an independent risk factor for disease-free survival in women with operable breast cancer. $B M C$ Cancer. 2004;4(1):82.

6. Menen RS, Hunt KK. Considerations for the treatment of young patients with breast cancer. Breast J. 2016;22(6):667-672.

7. Reyna C, Lee MC. Breast cancer in young women: special considerations in multidisciplinary care. J Multidiscip Healthc. 2014;7:419-429.

8. Azim HA, Nguyen B, Brohée S, Zoppoli G, Sotiriou C. Genomic aberrations in young and elderly breast cancer patients. BMC Med. 2015;13(1):266.

9. Howard-Anderson J, Ganz PA, Bower JE, Stanton AL. Quality of life, fertility concerns, and behavioral health outcomes in younger breast cancer survivors: a systematic review. J Natl Cancer Inst. 2012;104(5):386-405.

10. Arndt V, Merx H, Stürmer T, Stegmaier C, Ziegler H, Brenner H. Agespecific detriments to quality of life among breast cancer patients one year after diagnosis. Eur J Cancer. 2004;40(5):673-680.

11. Tiedtke C, de Rijk A, Dierckx de Casterlé B, Christiaens MR, Donceel P. Experiences and concerns about 'returning to work' for women breast cancer survivors: a literature review. Psychooncology. 2010;19(7):677-683.

12. Semple CJ, McCance T. Parents' experience of cancer who have young children: a literature review. Cancer Nurs. 2010;33(2):110-118.

13. Fobair P, Stewart SL, Chang S, D'Onofrio C, Banks PJ, Bloom JR. Body image and sexual problems in young women with breast cancer. Psychooncology. 2006;15(7):579-594.

14. Wright F, De Vito C, Langer B, Hunter A. Multidisciplinary Cancer Conference Standards. Toronto (ON): Cancer Care Ontario; 2006.

15. Prades J, Remue E, van Hoof E, Borras JM. Is it worth reorganising cancer services on the basis of multidisciplinary teams (MDTs)? A systematic review of the objectives and organisation of MDTs and their impact on patient outcomes. Health Policy. 2015;119(4):464-474.

16. Pillay B, Wootten AC, Crowe $\mathrm{H}$, et al. The impact of multidisciplinary team meetings on patient assessment, management and outcomes in oncology settings: A systematic review of the literature. Cancer Treat Rev. 2016;42:56-72.

17. Fleissig A, Jenkins V, Catt S, Fallowfield L. Multidisciplinary teams in cancer care: are they effective in the UK? Lancet Oncol. 2006;7(11):935-943.

18. Patkar V, Acosta D, Davidson T, Jones A, Fox J, Keshtgar M. Cancer multidisciplinary team meetings: evidence, challenges, and the role of clinical decision support technology. Int J Breast Cancer. 2011;2011:1-7.

19. Ruhstaller T, Roe H, Thürlimann B, Nicoll JJ. The multidisciplinary meeting: An indispensable aid to communication between different specialities. Eur J Cancer. 2006;42(15):2459-2462.

20. Newman EA, Guest AB, Helvie MA, et al. Changes in surgical management resulting from case review at a breast cancer multidisciplinary tumor board. Cancer. 2006;107(10):2346-2351.

21. Landercasper J, Attai D, Atisha D, et al. Toolbox to reduce lumpectomy reoperations and improve cosmetic outcome in breast cancer patients: The American Society of Breast Surgeons Consensus Conference. Ann Surg Oncol. 2015;22(10):3174-3183. 
22. Churilla TM, Egleston BL, Murphy CT, et al. Patterns of multidisciplinary care in the management of non-metastatic invasive breast cancer in the United States Medicare patient. Breast Cancer Res Treat. 2016;160(1):153-162.

23. Logan S, Perz J, Ussher J, Peate M, Anazodo A. Clinician provision of oncofertility support in cancer patients of a reproductive age: a systematic review. Psycho Oncol. 2018;27(2):401-409.

24. Graham ID, Logan J, Harrison MB, et al. Lost in knowledge translation: time for a map? J Contin Educ Health Prof. 2006;26(1):13-24.

25. Thomas DR. A general inductive approach for analyzing qualitative evaluation data. Am J Eval. 2006;27(2):237-246.

26. Lamb BW, Taylor C, Lamb JN, et al. Facilitators and barriers to teamworking and patient centeredness in multidisciplinary cancer teams findings of a national study. Ann Surg Oncol. 2013;20(5):1408-1416.

27. Walsh J, Harrison JD, Young JM, Butow PN, Solomon MJ, Masya L. What are the current barriers to effective cancer care coordination? A qualitative study. BMC Health Serv Res. 2010;10(1):132.

28. Lostumbo L, Carbine NE, Wallace J, Ezzo J, Dickersin K. Prophylactic mastectomy for the prevention of breast cancer. The Cochrane Library. 2004 volume 4.

29. Tuttle TM, Abbott A, Arrington A, Rueth N. The increasing use of prophylactic mastectomy in the prevention of breast cancer. Curr Oncol Rep. 2010;12(1):16-21.

30. Lamb BW, Brown KF, Nagpal K, Vincent C, Green JS, Sevdalis N. Quality of care management decisions by multidisciplinary cancer teams: a systematic review. Ann Surg Oncol. 2011;18(8):2116-2125.
31. Tripathy D. Multidisciplinary care for breast cancer: barriers and solutions. Breast J. 2003;9(1):60-63.

32. Look Hong NJ, Gagliardi AR, Bronskill SE, Paszat LF, Wright FC. Multidisciplinary cancer conferences: exploring obstacles and facilitators to their implementation. J Oncol Pract. 2010;6(2):61-68.

33. Hong NJ, Wright FC, Gagliardi AR, Paszat LF. Examining the potential relationship between multidisciplinary cancer care and patient survival: an international literature review. J Surg Oncol. 2010;102(2): 125-134.

34. Wright FC, de Vito C, Langer B, Hunter A, Expert Panel on Multidisciplinary Cancer Conference Standards. Multidisciplinary cancer conferences: a systematic review and development of practice standards. Eur J Cancer. 2007;43(6):1002-1010.

35. Brar SS, Hong NL, Wright FC. Multidisciplinary cancer care: does it improve outcomes? J Surg Oncol. 2014;110(5):494-499.

36. Marsh CJ, Boult M, Wang JX, Maddern GJ, Roder DM, Kollias J. National Breast Cancer Audit: the use of multidisciplinary care teams by breast surgeons in Australia and New Zealand. Med J Aust. 2008;188(7):385-388

37. Wilcoxon H, Luxford K, Saunders C, Peterson J, Zorbas H, on behalf of National Breast and Ovarian Cancer Centre's Multidisciplinary Care Audit Steering Committee. Multidisciplinary cancer care in Australia: A national audit highlights gaps in care and medico-legal risk for clinicians. Asia Pac J Clin Oncol. 2011;7(1):34-40.
Journal of Multidisciplinary Healthcare

\section{Publish your work in this journal}

The Journal of Multidisciplinary Healthcare is an international, peerreviewed open-access journal that aims to represent and publish research in healthcare areas delivered by practitioners of different disciplines. This includes studies and reviews conducted by multidisciplinary teams as well as research which evaluates the results or conduct of such teams or health

\section{Dovepress}

care processes in general. The journal covers a very wide range of areas and welcomes submissions from practitioners at all levels, from all over the world The manuscript management system is completely online and includes a very quick and fair peer-review system. Visit http://www.dovepress.com/ testimonials.php to read real quotes from published authors. 